\title{
Avaliação de indícios de TDAH por meio de três escalas
}

\author{
Evidence assessment of ADHD using three scales
}

Simone Chabudee Pylro ${ }^{[a]}$, Claudia Broetto Rossetti ${ }^{[b]}$

\footnotetext{
[a] Doutora em Psicologia pela Universidade Federal do Espírito Santo, professora da Universidade Vila Velha, Espírito Santo - Brasil, e-mail:

simone.chabudee@gmail.com

${ }^{[b]}[b]$ Doutora em Psicologia Escolar e do Desenvolvimento pela Universidade de São Paulo, professora da Graduação e do Programa de Pós-graduação em Psicologia da Universidade Federal do Espírito Santo, Vitória, Espírito Santo - Brasil, e-mail: cbroetto.ufes@gmail.com
}

Recebido: 09/05/2013 Received: 05/09/2013

Aprovado: 02/09/2013 Approved: 09/02/2013

\section{Resumo}

O Transtorno de Déficit de Atenção e Hiperatividade (TDAH) vem despertando o interesse de estudiosos, de modo que já estão disponíveis vários instrumentos que podem ser utilizados no seu diagnóstico, especialmente para o público infantil. Considerando a importância de melhor conhecer o uso de alguns desses instrumentos junto a adolescentes, este estudo buscou: (a) identificar, no contexto escolar, estudantes do Ensino Médio que apresentassem indícios de TDAH, por meio da aplicação da Escala para Transtorno de Déficit de Atenção/Hiperatividade - Versão para professores; (b) comparar os resultados obtidos na aplicação da referida escala com os obtidos por meio da aplicação dos questionários ASRS-18 aos adolescentes e SNAPIV aos pais. Para tal, participaram 12 estudantes de uma instituição pública de ensino, nove professores dos referidos alunos e 12 pais/responsáveis dos discentes participantes. Os dados obtidos pela comparação entre os três instrumentos corroboraram resultados já identificados em outros estudos, tais como a baixa concordância entre professores, pais e estudantes quanto aos sintomas de TDAH. Dadas as peculiaridades da avaliação em adolescentes, seria interessante investir-se em material específico para este público no que se refere às queixas de desatenção e hiperatividade. Por fim, vale lembrar que, tendo em vista que o número de participantes desse estudo foi restrito, os dados aqui relatados devem ser analisados com cautela e sem generalizações, havendo a necessidade de pesquisas futuras que possam explorar melhor tais questões.

Palavras-chave: Transtorno do Déficit de Atenção com Hiperatividade. Avaliação. Escalas. Psicologia.

\section{Abstract}

The Attention Deficit Hyperactivity Disorder (ADHD) has aroused the interest of scholars, so that there are several tools already available that can be used to diagnose it, especially for children. Considering the importance of better understanding the use of these instruments with adolescents, this study has attempted to: (a) identify, in the school context, high school students showing evidence of ADHD, through the application of the Scale for Attention Deficit Disorder/Hyperactivity Disorder-Version for teachers; (b) compare the results obtained from the application of the scale to those obtained through the application of the ASRS-18 questionnaire with the adolescents and 
the SNAP-IV, with their parents. To this end, 12 students from public school participated, as well as nine teachers of these students and 12 parents/guardians of them. The data obtained from the comparison between the three instruments corroborated results already identified in other studies, such as the low agreement between teachers, parents and students about the symptoms of ADHD. Given the peculiarities of the assessment in adolescents, it would be interesting to invest in specific material for this audience regarding the complaints of inattention and hyperactivity. Finally, it is worth noting that the number of participants in this study was limited, therefore the data reported should be interpreted with caution and without generalizations, with the need for future research that could further explore such issues.

Keywords: Attention Deficit Hyperactivity Disorder. Assessment. Scales. Psychology.

\section{Introdução}

Inicialmente nomeado "Síndrome Hipercinética", depois "lesão cerebral mínima" (1947) e "disfunção cerebral mínima" (1962), 0 Transtorno de Déficit de Atenção e Hiperatividade (TDAH) é um quadro neurobiológico caracterizado pelo "desempenho inapropriado dos mecanismos que regulam a atenção, a reflexibilidade e a atividade, sendo, portanto, um transtorno heterogêneo, de instalação na infância e que se caracteriza pela desatenção, impulsividade e hiperatividade" (Andrade \& Morais, 2006, p. 136).

Quanto aos critérios diagnósticos para este transtorno, duas referências principais têm sido amplamente utilizadas: a Classificação Internacional de Doenças (CID), proposta pela Organização Mundial de Saúde (OMS), e o Manual Diagnóstico de Doenças Mentais (DSM), apresentado pela Associação Americana de Psiquiatria (APA).

Na CID-10, a nomenclatura utilizada é Transtorno Hipercinético, cuja definição prevê a existência de seis ou mais sintomas de desatenção, três ou mais sintomas de hiperatividade e um sintoma de impulsividade. 0 diagnóstico prevê a presença destas três categorias de sintomas em diferentes lugares e ocasiões.

Em 1994, o TDAH foi definido mais claramente no DSM-IV, de modo que a impulsividade também passou a ter relevância na descrição desse transtorno (Rotta, Ohlweiler \& Riesgo, 2006; Rossi, 2008). Segundo o manual, o TDAH abrange três subtipos: predominantemente hiperativo-impulsivo, predominantemente desatento e combinado ou misto. Contudo, estudiosos defendem que o mais importante no diagnóstico é verificar o grau de prejuízo para a vida dos pacientes, uma vez detectados os referidos sintomas (Rohde, Barbosa, Tramontina \& Polanczyk, 2000).

A literatura sobre o TDAH tem apontado que o transtorno é diagnosticado com maior incidência em meninos do que em meninas, estabelecendo uma razão entre o sexo masculino e feminino de até 9:1. No entanto, apesar de ser comum considerar-se o TDAH uma doença tipicamente masculina, "os dados encontrados sobre a prevalência do TDAH variam em grande escala em função da metodologia, do contexto em que ocorrem os estudos (amostra clínica ou de comunidade), da população estudada e da fonte dos dados das amostras" (Gobbo \& Netto, 2010, p. 226-227). Além disso, alguns autores discutem que há uma subidentificação e subdiagnosticação das meninas (Asbahr, Costa \& Morikawa, 2010, Biederman et al., 2002). Uma análise recorrente sobre esta questão é que tal fato ocorra devido aos pais e professores darem maior importância aos sintomas externalizantes, mais comuns entre os meninos, do que aos sintomas internalizantes, mais recorrentes em meninas.

O TDAH vem despertando o interesse de estudiosos e do público em geral, de maneira que já é considerado por alguns como o tipo de desordem comportamental de caráter crônico que mais aparece na infância, e o mais estudado (Vasconcelos et al., 2005; Schneider \& Eisenberg, 2006; Peixoto, 2006).

No que se refere aos adolescentes e aos adultos, segundo o DSM-IV,

os sintomas de hiperatividade assumem a forma de sentimentos de inquietação e dificuldade para envolver-se em atividades tranquilas e sedentárias. A impulsividade manifesta-se como impaciência, dificuldade para protelar respostas, 
responder precipitadamente, antes do término das perguntas (Critério A2g), dificuldade para aguardar sua vez (Critério A2h) e interrupção frequente ou intromissão nos assuntos alheios, ao ponto de causar dificuldades em contextos sociais, escolares ou profissionais (Critério A2i). Outros podem queixar-se de dificuldade para se expressar adequadamente. Os indivíduos com este transtorno tipicamente fazem comentários inoportunos, não obedecem a instruções, iniciam conversas em momentos inoportunos, interrompem demais os outros, intrometem-se em assuntos alheios, agarram objetos de outros, pegam coisas que não deveriam tocar e fazem palhaçadas (APA, 2002, p.113).

A popularização do TDAH se evidencia pelo espaço que o tema tem ocupado na mídia e no interesse da sociedade como um todo. A busca pelo assunto em sites da Internet, ou mesmo em bases exclusivamente científicas, permite perceber como o TDAH tem sido apresentado associado aos mais variados problemas: dificuldades de aprendizagem, dificuldades de relacionamento social, violência, determinação genética, drogas, entre outros.

Apesar do caráter contemporâneo desta temática, o início da discussão sobre hiperatividade e desatenção data do Século XVIII (Folquito, 2009). Porém, nos últimos 30 anos, o interesse pelo assunto cresceu muito, de maneira que assistimos, nas décadas de 80 e 90 do século passado, a um boom publicitário em torno do TDAH e da Ritalina, um dos medicamentos mais divulgados no tratamento do transtorno (Eberstadt, 1999; Caliman, 2008), tornando-os cada vez mais conhecidos. Quanto ao uso de medicamentos no tratamento do TDAH no Brasil, é inegável que houve um crescimento vertiginoso, o que pode ser confirmado, por exemplo, por índices apresentados em uma sessão divulgada no Jornal do Conselho Federal de Psicologia (CFP) no mês de Outubro de 2011:

em oito anos, aumentou $1.616 \%$ o consumo de Metilfenidato, princípio ativo dos remédios Ritalina e Concerta, receitados para crianças com hiperatividade ou déficit de atenção. Em 2000, foram vendidas 71 mil caixas desses medicamentos no Brasil. Em 2008, as vendas atingiram 1,14 milhão (CFP, 2011, p.8).
Essa situação tem provocado o debate de profissionais da área que discutem a complexidade e multifatorialidade da vida e da doença, e que por vezes veem questões de convivência e inadequação tratadas em um espectro reducionista, como problemas exclusivamente médicos, sem o cuidado de buscar um tratamento interdisciplinar.

Assim, aspectos ligados ao diagnóstico do TDAH têm sido alvo de críticas dado seu caráter controverso, sendo encontrado, essencialmente, a partir de uma avaliação clínica pautada na análise de uma sintomatologia comportamental respaldada em critérios propostos em manuais (Folquitto, 2009; Caliman, 2008; Mattos et al., 2006); e cujos estudos ainda estão muito circunscritos ao campo da medicina e áreas afins, de modo que a maioria das explicações para a compreensão do fenômeno ainda situam-se prioritariamente no campo biológico.

Segundo Rohde et al. (2000), a prevalência do TDAH situa-se em torno de $3 \%$ e $6 \%$ entre crianças em idade escolar e caracteriza-se pela tríade desatenção, hiperatividade e impulsividade. A lista de comportamentos que se encaixam nesses três aspectos é extensa, de modo que uma gama muito grande de características cabe no diagnóstico desse transtorno, que acaba funcionando como uma espécie de "para-raios". Além disso, outro aspecto que acirra a discussão entre os estudiosos do tema é o fato de que alguns outros quadros patológicos apresentados no DSM IV, também indicam a manifestação de problemas relacionados à desatenção, concentração ou impulsividade (Caliman, 2008).

Tal situação dificulta o estabelecimento de um consenso e clareza na descrição do TDAH. Ainda sobre questões relativas ao diagnóstico,

apesar dos avanços dos métodos de visualização cerebral, no dia-a-dia da prática diagnóstica eles não revelam muita coisa. Até o momento, nenhum teste ou exame específico e preciso para a "identificação" do TDAH foi definido. Seu diagnóstico continua sendo feito através de um processo misto, que inclui testes psicológicos, história clínica, análise do desempenho escolar e entrevistas com pais e professores (Caliman, 2008).

0 processo histórico que levou à construção da concepção do TDAH como um transtorno se deve, 
em parte, exatamente à dificuldade de efetuar-se um diagnóstico mais preciso. Por isso, com relação ao Déficit de Atenção e Hiperatividade, é mais prudente utilizar a expressão transtorno, tendo em vista as manifestações comportamentais presentes, "do que propriamente uma disfunção, já que o que se observa (...) necessariamente, é uma alteração no comportamento e na capacidade de manter a atenção" (Folquitto, 2009, p. 19).

Com o reconhecimento do TDAH como um transtorno, oficializado no DSM IV, o reconhecimento de sua importância foi crescente e, em algumas sociedades, chegou ao ponto de ocupar grande relevância social. Um exemplo disso pode ser verificado nos Estados Unidos da América (EUA), no movimento que instituiu sua entrada nas datas oficiais, criando o "Dia da Consciência Nacional sobre o TDAH" (Caliman, 2008; EUA, 2004).

Nesse processo de investigação e popularização, a abrangência do tema também foi se ampliando, de modo que o adolescente e o adulto com sintomas de TDAH passaram a compor a lista de interesses sobre a questão, levando à adoção de uma perspectiva de desordem do desenvolvimento que se mantém na vida adulta, não sendo apenas exclusividade da infância (Caliman, 2008).

Apesar da tendência em se afirmar que o TDAH persiste ao longo da vida, estudiosos da área concordam que os sintomas do TDAH podem diminuir com a idade (Biederman, Mick \& Faraone, 2000; Achenbach, Howell, Mcconaughy \& Stanger, 1998), de modo que no curso do desenvolvimento é possível verificar uma diminuição da hiperatividade, restando com frequência desatenção e impulsividade, especialmente cognitiva, tais como agir antes de pensar (Rohde \& Halpern, 2004).

Considerando a dificuldade em diagnosticar o Transtorno de Déficit de Atenção e Hiperatividade, já descritas anteriormente, e a necessidade de critérios mais objetivos para auxiliar na avaliação, especialistas da área têm proposto instrumentos pautados em critérios diagnósticos da CID-10 do DSM-IV. Tais instrumentos, em geral, são aplicados junto aos professores, aos pais e à própria pessoa sobre quem há suspeitas de estar manifestando os sintomas de TDAH.

Segundo Antoniuk, (2006), entre os principais instrumentos utilizados no auxílio do diagnóstico pode-se citar o CBCL (Child Behavior
Checklist), a escala ADHD RS-IV (Attention Deficit/ Hyperactivity Disorder Rating Scale-IV), a escala de Conners, o Questionário de Swanson, Nolan e Pelham-IV (SNAP IV), a EDAH (Escalas para la Evaluación del Transtorno por Déficit de Atención con Hiperactividad), na Espanha, e a Escala de Transtorno de Déficit de Atenção/Hiperatividade (Benczick, 2000a), no Brasil.

Tais instrumentos podem ser utilizados como recurso auxiliar no diagnóstico, como também podem ser aplicados no monitoramento da evolução clínica do transtorno. Entretanto, Antonuik (2006) destaca que são poucos os "estudos de confiabilidade, validade e padronização das referidas escalas no Brasil" (p. 3).

Com o intuito de conhecer quais destes instrumentos estão sendo mais utilizados no contexto de pesquisa no Brasil, e considerando que tal aproximação parece ser possível por meio de uma busca no Banco de Teses e Dissertações da CAPES, foi realizado um levantamento a este respeito. A busca foi inspirada nos instrumentos mencionados por Antoniuk, (2006). Vale ressaltar que nesse levantamento decidimos excluir o CBCL (Child Behavior Checklist) - um questionário aplicado aos pais que pretende aferir quesitos relacionados à competência social e problemas comportamentais em pessoas com idade entre 04 e 18 anos, assim como a Escala de Conners, por serem instrumentos de diagnóstico de amplo espectro, não se limitando ao diagnóstico do TDAH (Graeff \& Vaz, 2008). Além disso, incluímos palavras que fizessem menção à Adult Self-Report Scale (ASRS), tendo em vista que tínhamos a intenção de usar esse instrumento no presente estudo. A seleção dos trabalhos foi feita a partir do campo "assunto" do site de busca, disponibilizado pelo Banco de Teses da CAPES, no período referente a 1987-2010, com os seguintes descritores: SNAP-IV, ASRS, Adult Self Report Scale, Benczik, TDAH + versão para professores, ADHD RS-IV, Attention Deficit/Hyperactivity Disorder Rating Scale-IV, EDAH, Escalas para la Evaluación del Transtorno por Déficit de Atención com Hiperactividad. Tal busca revelou um total de 39 trabalhos.

Após este primeiro levantamento, filtramos os trabalhos em que o TDAH era o foco central do estudo, o que nos permitiu identificar 23 pesquisas. Destas, 12 foram realizadas com crianças; 06 com crianças e 
adolescentes; 01 com adolescentes; 02 com adultos; 02 com crianças, adolescentes e adultos ${ }^{1}$.

A partir dessas reflexões sobre as dificuldades no diagnóstico do TDAH e, tendo em vista que a quantidade de estudos voltados para adolescentes ainda é inferior à produção relativa à infância, buscou-se (a) Identificar, no contexto escolar, estudantes do Ensino Médio que apresentassem indícios de TDAH, e estudantes que não apresentam tais indícios; (b) Comparar os resultados obtidos na aplicação da Escala para Transtorno de Déficit de Atenção/Hiperatividade - Versão para professores (Benczik, 2000a) e dos Questionário ASRS-18 (Mattos, Segenreich, Saboya, Louzã, Dias \& Romano, 2006) ${ }^{2}$ e SNAP-IV (Kessler et. al., 2005) ${ }^{3}$ quanto aos sinais de TDAH.

É importante destacar que a presente pesquisa faz parte de um recorte de um levantamento mais amplo (Pylro, 2012), na qual se buscou identificar, no contexto escolar, estudantes do Ensino Médio que apresentassem indícios de TDAH. Para sua composição, procurou-se comparar os resultados obtidos na aplicação das três escalas indicadas acima nos participantes, seus professores e pais.

No que diz respeito aos cuidados éticos, este estudo foi aprovado pelo Comitê de Ética em Pesquisa do Centro de Ciências da Saúde da Universidade Federal do Espírito Santo, sob o protocolo ${ }^{\circ} 215 / 09$ de acordo com as Resoluções 196/96, 251/97 e 292/99 do Conselho Nacional de Saúde que preconiza as normas de pesquisas envolvendo seres humanos.

\section{Método}

\section{Participantes e local de coleta de dados}

Inicialmente foram aplicadas 43 Escalas de TDAH - versão para professores (Benczik, 2000a), sendo que nove professores participaram respondendo-as. Houve a necessidade de se descartar 18 escalas

1 As referências completas dos trabalhos podem ser encontradas em (2012).

2 Disponível em http://www.tdah.org.br/br/sobre-tdah/ diagnostico-adultos.html

3 Disponível em http://www.hcp.med.harvard.edu/ncs/ asrs.php por conta da desistência de alguns participantes ou por estes não se enquadrarem nos parâmetros propostos pela escala (idade máxima de 17 anos, por exemplo). Além desse descarte inicial, os dados de um dos participantes tiveram que ser deixados de lado por conta de sua desistência no decorrer da coleta. Os estudantes com indícios de TDAH, assim como os pais/ responsáveis destes, também participaram respondendo, respectivamente, aos questionários ASRS 18 e SNAP-IV.

Assim, os dados apresentados a seguir dizem respeito aos 12 estudantes que, após dados coletados por meio da Escala para Transtorno de Déficit de Atenção/Hiperatividade - Versão para professores (Benczik, 2000a), apresentaram indícios de TDAH, sendo todos do $2^{\circ}$ ano de uma mesma escola estadual de Ensino Médio. A escola em questão pertence à rede pública do Estado do Espírito Santo, e os professores participantes trabalhavam com as disciplinas de Biologia, Educação Física, História, Matemática e Português. A inclusão desses professores se deu prioritariamente por sua disponibilidade e pelo grau de conhecimento dos alunos participantes, uma vez que teriam que julgar quesitos específicos sobre seu desempenho no contexto escolar.

Instrumentos

a) Para a coleta de dados foram utilizados os seguintes instrumentos:

b) Escala para Transtorno de Déficit de Atenção/Hiperatividade - Versão para professores (Benczik, 2000) - respondida pelos professores;

c) Adult Self-Report Scale (ASRS 18) - Optamos por aplicar este instrumento junto aos adolescentes, tendo em vista que à época da coleta tivemos dificuldade em encontrar material específico para o público adolescente. Consideramos que os tópicos presentes no questionário eram possíveis de serem respondidos pelo próprio estudante, considerando o perfil e a faixa etária dos adolescentes com os quais trabalhamos;

d) Questionário SNAP-IV (Mattos, SerraPinheiro, Rohde \& Pinto, 2006) - respondido pelos pais. 


\section{Procedimentos}

Mediante autorização da escola, conversa com uma das pedagogas e com os professores, foram indicados alunos do $2^{\circ}$ ano do Ensino Médio que, segundo a perspectiva da escola, apresentavam indícios de TDAH. Após explicação dos procedimentos e assinatura do Termo de Consentimento Livre e Esclarecido, passou-se à aplicação da Escala para Transtorno de Déficit de Atenção/Hiperatividade - Versão para professores (Benczik, 2000a), que permitiu levantar, dentre os alunos listados, 12 estudantes com indícios de TDAH. A referida escala foi aplicada individualmente junto aos professores, em horários previamente combinados, na própria escola. Em seguida, após os devidos esclarecimentos junto aos estudantes e a sua anuência em participar, o que pôde ser atestado mediante a assinatura de um termo de assentimento, encaminhamos uma carta explicativa aos pais/responsáveis acompanhada de um termo de autorização para aqueles que concordassem com que seus filhos participassem do estudo. 0 referido termo foi encaminhado junto à uma cópia do Questionário SNAP-IV, respondido pelos pais sem a supervisão da pesquisadora. O questionário ASRS-18 só foi aplicado junto aos estudantes depois da autorização dos pais e da devolução do questionário SNAP-IV. A coleta de dados junto aos adolescentes foi realizada individualmente, na própria instituição de ensino, em salas de aula desocupadas indicadas pela escola. As respostas dos adolescentes ao Questionário ASRS-18, além de terem sido registradas em protocolo próprio, também foram gravadas digitalmente, após o consentimento destes.

\section{Resultados e discussão}

Coletar dados em escola é uma tarefa difícil, pois somos desafiados a interferir o mínimo possível na rotina da escola para não haver prejuízo nos processos ali desenvolvidos e, ao mesmo tempo, conciliar horários comuns em agendas muito corridas, como é o caso de professores que trabalham em dois ou três turnos, dispondo de pouco ou nenhum tempo. Assim, em primeiro lugar, é importante ressaltar que a coleta só foi possível em razão do esforço e da colaboração dos funcionários da escola, o que incluiu professores, coordenadores, supervisores, equipe da secretaria e equipe de apoio, em especial da supervisora do turno da tarde, que sempre se colocou de forma muito colaborativa. Entre as principais dificuldades encontradas na aplicação da Escala para Transtorno de Déficit de Atenção/ Hiperatividade - Versão para professores (Benczik, 2000a), destacamos: (a) a falta de tempo dos professores para responder ao instrumento, o que fez com que esta etapa da coleta tivesse que ser feita prioritariamente nos horários de planejamento (comumente chamados de PL) dos participantes, em sua maioria na sala dos professores; (b) a falta de contato mais próximo dos professores com os alunos, restringindo quais docentes poderiam responder à escala e revelando dificuldade por parte de alguns professores em responder a alguns itens do instrumento com maior precisão.

Apesar de o TDAH ser um termo popular na área da educação, o conhecimento sobre a sintomatologia desse transtorno parece ainda não ter sido divulgado o suficiente para professores brasileiros. Esta parece ser uma situação relevante, considerando a importância do relato desses profissionais para a composição do diagnóstico do TDAH (Coutinho, Mattos, Schmitz, Fortes, \& Borges, 2009).

Segundo Benczik (2000b), autora da escala que usamos junto aos professores, o ideal é que, quando o aluno tem vários professores, todos respondam e se faça uma média dos resultados. Porém, diante das dificuldades já mencionadas, não foi possível atender a este critério.

Vencidos estes desafios, após a aplicação da Escala para Transtorno de Déficit de Atenção/ Hiperatividade - Versão para professores (Benczik, 2000a), verificou-se que, segundo a percepção dos professores participantes, 12 estudantes, nove do sexo feminino e três do sexo masculino, denotaram indícios de TDAH, sendo que tais indícios configuraram-se conforme descrição a seguir Déficit de Atenção: 11 (84,6\%), Hiperatividade: 8 (61,5\%), Problemas de Aprendizagem: 4 (30,7\%), Comportamento antissocial, $4(30,7 \%)$.

Um dado curioso, que logo pode despertar a atenção do leitor, é o fato de nossa amostra revelar um predomínio do sexo feminino, o que não é usual encontrar nos estudos sobre o tema. A literatura, porém, também assinala que aspectos metodológicos e contextuais, assim como a valorização de determinados sintomas em detrimento de outros, podem explicar a situação (Gobbo \& Neto, 2010; Asbahr et al., 2010, 
Biederman et al., 2002). Deve-se destacar, ainda, o fato de não termos tido a pretensão de fazer um levantamento da incidência do TDAH junto à população de alunos do $2^{\mathrm{a}}$ ano na escola em que realizamos a coleta. Levantamos apenas alguns casos que os professores julgaram apresentar indícios do transtorno.

Quanto à apresentação dos sintomas, observou-se um predomínio do Déficit de Atenção e da Hiperatividade, respectivamente. Contudo, dentre os 12 estudantes, oito apresentaram indícios em mais de um dos quesitos avaliados pela escala, sendo que as combinações mais frequentes foram Déficit de Atenção + Hiperatividade + Comportamento Antissocial e Déficit de Atenção + Hiperatividade + Problemas de Aprendizagem.

Quanto aos indicativos diagnósticos, encontramos uma distribuição parecida com a apresentada por Biederman et. al. (2002), de modo que houve uma maior incidência do subtipo combinado, seguido pelo desatento e depois o impulsivo, conforme se pode observar na tabela a seguir.

Tabela 1 - Característica da amostra quanto aos quesitos Déficit de Atenção Hiperatividade, Problemas de Aprendizagem, Comportamento Antissocial

\begin{tabular}{lcc}
\hline Característica & Participante & n (\%) \\
\hline $\begin{array}{l}\text { Déficit de Atenção } \\
\text { Hiperatividade }\end{array}$ & $01 / 03 / 09 / 13$ & $4(30,7 \%)$ \\
$\begin{array}{l}\text { Déficit de Atenção + } \\
\text { Hiperatividade } \\
\text { Hiperatividade + } \\
\text { Comportamento Antissocial }\end{array}$ & 08 & $1(7,7 \%)$ \\
$\begin{array}{l}\text { Déficit de Atenção + } \\
\text { Probl. de Aprendizagem }\end{array}$ & 12 & $1(7,7 \%)$ \\
$\begin{array}{l}\text { Déficit de Atenção + } \\
\text { Hiperat. + Comp. Antiss. }\end{array}$ & 07 & $1(7,7 \%)$ \\
$\begin{array}{l}\text { Déficit de Atenção + } \\
\text { Hiperat. + Probl. de Aprend. }\end{array}$ & $02 / 04$ & $2(15,4 \%)$ \\
$\begin{array}{l}\text { Déficit de Atenção + Hiperat. } \\
+ \text { Probl. de Aprendizagem + } \\
\text { Comportamento Antissocial }\end{array}$ & 05 & $1(7,7 \%)$ \\
\hline
\end{tabular}

Apesar de algumas pesquisas indicarem que há uma maior chance de encontrar-se o subtipo desatento no sexo feminino, Gobbo e Neto (2010) discutem que os sintomas de hiperatividade e impulsividade, associados comumente aos meninos, também aparecem, em estudos mais recentes, nas meninas (Staller \& Faraone, 2006, citados por Gobbo \& Neto, 2010), não denotando muitas diferenças clínicas no que se refere ao sexo. Quando são observadas distinções, é possível que ocorram em função de algum viés metodológico (Biederman et al., 2002, citado por Gobbo \& Neto, 2010).

A coleta junto aos alunos ocorreu individualmente, dentro da própria instituição de ensino, em horários estipulados por esta que não comprometessem a rotina do discente. A etapa foi realizada em salas de aula, determinadas pela escola, vazias no momento da aplicação. Entregamos o questionário ao participante para que ele marcasse a opção adequada em cada pergunta mas, ao mesmo tempo, gravamos as verbalizações proferidas ao longo da aplicação, o que muito enriqueceu o processo de análise.

Ao aplicarmos o Questionário ASRS-18 nos alunos para avaliarmos sua percepção quanto aos indícios mencionados pelos professores, verificamos que, entre os 12 alunos indicados, cinco, ou seja, 41,7\%, se autoavaliaram com algum indício de TDAH.

Quanto à coerência entre a autopercepção dos estudantes e a avaliação dos professores sobre os quesitos desatenção e hiperatividade, houve aproximação exceto quanto a dois dos participantes. Um desses participantes não se considerou hiperativo e comentou acerca de uma possível agitação sua no contexto escolar: "[a agitação] depende do local. É onde se sente mais a vontade. Há locais em que não se pode. Com os colegas da escola fica mais a vontade e exibe esses comportamentos de agitação, com outras pessoas se controla mais."

Já o outro estudante, que também não se avaliou como hiperativo, afirmou: "Eu consigo ficar [sossegada], mas não por muito tempo". Ao falar sobre seu comportamento desatento, explica:

Eu tenho que gostar, se eu gostar, se eu entender aí eu presto atenção; agora se eu não gostar e se eu achar muito chato, eu não consigo prestar atenção. Se for difícil aí eu tento prestar atenção, aí eu presto atenção mais, para entender cada vez mais. Ah, não sei, às vezes eu sou muito impaciente e quando eu não estou a fim mesmo de prestar atenção, eu não consigo, eu começo a falar, a conversar, mas quando eu quero prestar atenção eu presto; agora, quando eu não quero, não tem jeito, não consigo. É, é quando eu não tenho interesse, quando eu 
olho e não me interessa, aí eu não consigo prestar atenção.

A fala desses estudantes parece indicar que, além de possíveis suspeitas quanto a sintomas do TDAH, há que se avaliar outras circunstâncias que atravessam o processo de escolarização e envolvem experiências comuns nesta fase da vida, como a motivação e o interesse do aluno frente a atividades escolares, as metodologias empregadas, etc. Tais situações podem levar o aluno a posturas de desinteresse ou mesmo desatenção. Além disso, é comum que no ambiente escolar se estabeleça um clima de amizade e proximidade que deixe o aluno mais à vontade em comparação a outros espaços.

Tivemos alguns problemas na aplicação do questionário SNAP-IV aos pais, de modo que três questionários retornaram sem o preenchimento de uma parte final em que constavam perguntas sobre quando os sintomas tiveram início e os contextos em que evidenciavam-se. Também verificamos que a avaliação dos pais quanto aos indícios de TDAH foi bem diversa do julgamento feito pelos professores e da autoavaliação dos alunos. Assim, segundo a percepção dos pais, apenas um aluno foi avaliado como tendo indícios de desatenção ou hiperatividade.

Dentre as possíveis análises que podem ter influenciado a resposta dos pais ou responsáveis, destacamos (a) que alguns deles podem não ter um conhecimento mais próximo do dia a dia de seus filhos, em especial por se tratar de estudantes do Ensino Médio, o que dificultaria o preenchimento do instrumento; (b) que alguns podem ter tido uma postura leviana, de maneira que responderam rápida e incompletamente, procurando apenas dar conta da tarefa; (c) a probabilidade de alguns terem dificuldades com a leitura e escrita, comprometendo a compreensão dos itens do instrumento. Inicialmente, também consideramos a possibilidade de o próprio aluno ter respondido. Contudo, dado o caráter pouco coerente entre as informações levantadas no instrumento aplicado junto aos alunos e no direcionado aos pais, achamos essa última hipótese menos provável.

A tabela a seguir apresenta, resumidamente, uma comparação entre os resultados obtidos, após a aplicação dos três instrumentos, em relação à presença ou ausência de sintomas característicos do TDAH.

Sobre esse tipo de comparação Coutinho et al. (2009) comentam:

adolescentes tendem a subestimar seus sintomas de TDAH; pais geralmente são questionados sobre a manifestação de sintomas de TDAH não apenas em casa, mas também no ambiente escolar. A obtenção de informações utilizando apenas pais ou professores modifica significativamente a prevalência de TDAH. Professores frequentemente são os primeiros a sugerir que existe possível TDAH em crianças e adolescentes em idades escolares, reforçando a importância de relato proveniente da escola para

Tabela 2 - Comparação entre os resultados obtidos na aplicação da Escala de TDAH para Professores e os Questionários ASRS-18 e SNAP-IV

\begin{tabular}{|c|c|c|c|c|c|c|}
\hline \multirow{2}{*}{$\begin{array}{l}\text { Participante } \\
\text { Carla }\end{array}$} & \multicolumn{4}{|c|}{ Escala de TDAH para Prof. (BENCZIK) (percentil) } & \multirow{2}{*}{$\begin{array}{c}\text { Questionário ASRS-18 } \\
\text { Sem indícios }\end{array}$} & \multirow{2}{*}{$\frac{\text { Questionário SNAP-IV }}{\text { Sem indícios }}$} \\
\hline & $\mathrm{A}(85)$ & $\mathrm{B}(01)$ & $\mathrm{C}(75)$ & $\mathrm{D}(35)$ & & \\
\hline Camila & $A(99)$ & $\mathrm{B}(95)$ & $\mathrm{C}(95)$ & $\mathrm{D}(40)$ & Sem indícios & Sem indícios \\
\hline Caio & $A(95)$ & $\mathrm{B}(90)$ & $\mathrm{C}(85)$ & $\mathrm{D}(50)$ & Com indícios & Sem indícios \\
\hline Cristina & $A(95)$ & $\mathrm{B}(99)$ & $\mathrm{C}(80)$ & $\mathrm{D}(95)$ & Com indícios & Sem indícios \\
\hline Carina & $\mathrm{A}(85)$ & $\mathrm{B}(85)$ & $\mathrm{C}(65)$ & $\mathrm{D}(80)$ & Com indícios & Sem indícios \\
\hline César & $\mathrm{A}(85)$ & $\mathrm{B}(70)$ & $\mathrm{C}(80)$ & $\mathrm{D}(70)$ & Sem indícios & Com indícios \\
\hline Catarina & $A(75)$ & $\mathrm{B}(80)$ & $\mathrm{C}(30)$ & $\mathrm{D}(75)$ & Sem indícios & Sem indícios \\
\hline Clara & $\mathrm{A}(85)$ & $\mathrm{B}(25)$ & $\mathrm{C}(55)$ & $\mathrm{D}(40)$ & Sem indícios & Sem indícios \\
\hline Carolina & $A(75)$ & $\mathrm{B}(90)$ & $\mathrm{C}(25)$ & $\mathrm{D}(85)$ & Com indícios & Sem indícios \\
\hline Cíntia & $\mathrm{A}(85)$ & $\mathrm{B}(80)$ & $\mathrm{C}(40)$ & $\mathrm{D}(80)$ & Com indícios & Sem indícios \\
\hline Cauã & $\mathrm{A}(90)$ & $\mathrm{B}(85)$ & $\mathrm{C}(40)$ & $\mathrm{D}(75)$ & Sem indícios & Sem indícios \\
\hline Cátia & $\mathrm{A}(85)$ & $\mathrm{B}(55)$ & $\mathrm{C}(75)$ & $\mathrm{D}(75)$ & Sem indícios & Sem indícios \\
\hline
\end{tabular}

Legenda: A=Défic. Atenção; B=Hiper.; C=Probl. de Aprend.; D=Comp. Antissocial

Nota: Percentil $\geq 76 \rightarrow$ indícios de TDAH

Fonte: as autoras 
tal diagnóstico. Alguns estudos demonstraram que a correlação entre relatos de pais e professores é apenas modesta, e informantes apresentam melhor relato quando se referem a comportamentos restritos a seu ambiente de origem (isto e, pais relatam sobre comportamento em casa e professores relatam sobre comportamento na escola). Mais ainda, crianças com TDAH frequentemente apresentam comportamentos distintos, de acordo com o ambiente em questão (escola ou casa), o que sugere que relatos de pais acerca de comportamento na escola podem ser pouco precisos (p. 98).

A discrepância entre as respostas dos professores, pais e estudantes às escalas ou questionários leva à variação de prevalência de acordo com o informante (Golfeto \& Barbosa, 2003). Tal inconsistência é discutida por Mesquita (2009) e revela, segundo Cypel (2001), certa subjetividade na avaliação. Assim, "o que é considerado comportamento inadequado para um, pode ter pouco importância para outro" (pág. 34).

Segundo Benczik (2000a), os docentes tendem a superestimar os índicos de presença do TDAH, enquanto os pais e a própria criança ou adolescente tendem a subestimá-los.

Sobre a avaliação dos professores, Reinhardt (2007) comenta que as informações quanto aos sintomas do TDAH obtidas pelo docente, no que se refere aos estudantes adolescentes, são menos válidas. Isso porque nesta etapa do processo de escolarização o número de professores aumenta e diminui o tempo que passam com o aluno, prejudicando os meios do docente avaliá-lo.

\section{Considerações finais}

A comparação realizada entre as três escalas evidenciou dificuldades já identificadas em outros estudos que buscaram usar instrumentos para aferição de sintomas de TDAH.

Mesmo em pesquisas em que se aplicou o mesmo instrumento para pais e professores, foi encontrada baixa concordância em aspectos comportamentais. Para Andrade e Flores-Mendoza (2010), tal situação explica-se pelo conhecimento diferenciado dos docentes e dos pais acerca dos itens avaliados pelas escalas e questionários utilizados.

Diante deste fato, entendemos ser pertinente, como afirmam Coutinho et al. (2009), capacitar profissionais da área da educação acerca do TDAH, considerando a importância das informações por eles relatadas no diagnóstico do transtorno, especialmente no caso de crianças.

No que se refere aos adolescentes a situação parece ser um pouco mais complexa, dada as especificidades da escolarização nesta fase que incluem grande variedade de professores e pouca informação dos pais acerca de aspectos comportamentais e da rotina do adolescente na escola. Assim, seria interessante investir em material mais específico para este público.

0 cuidado com essas questões é de suma importância, pois os possíveis prejuízos em função de falsos diagnósticos podem ser bastante sérios (Carreiro et al., 2008). Assim, uma das possíveis ações para se minimizar o risco de falsos positivos ou negativos é a ação interdisciplinar. Ou seja, "à medida que a atuação profissional tiver um modo de interação que permita passar de um "fazer junto" para um "fazer conjunto", será possível produzir um conhecimento e ter uma intervenção mais eficaz, o que pode ser compreendido como a construção da interdisciplinaridade" (Carreiro et al., 2008, p. 65).

Por fim, vale lembrar que, tendo em vista a restrição do número de participantes deste estudo, os dados relatados devem ser analisados com cautela e sem generalizações, havendo a necessidade de pesquisas futuras que possam explorar melhor tais questões.

\section{Referências bibliográficas}

A crescente medicalização de esferas da vida preocupa a Psicologia. (2011). [Jornal do Federal] Jornal do Conselho Federal de Psicologia: Drogas: usuário precisa de cuidado, atenção e dignidade, ano XXIII (102) . Recuperado em 20 de março de 2012, de http://www. pol.org.br/pol/export/sites/default/pol/publicacoes/ publicacoesDocumentos/Jornal_Federal___final.pdf

Achenbach, T. M., Howell, C., Mcconaughy S., \& Stanger, C. (1998). Six year predictors of problems in a national sample, IV: young adult signs of disturbance. Journal of the American Academy of Child and Adolescent Psychiatry, v. 37 (7), 718-727.

American Psychiatric Association. (2002). Manual diagnóstico e estatístico de transtornos mentais. Porto Alegre: Artmed. 
Andrade, A. C., \& Flores-Mendoza, C. (2010). Transtorno do Déficit de Atenção/Hiperatividade: o que nos informa a investigação dimensional? Estudos de Psicologia, v. 15 (1), 17-24.

Andrade, E. R., \& Morais, R. M. C. B. (2006). Transtorno de déficit de atenção/hiperatividade. In: Abreu, C. N. de, Salzano, F. T., Vasques, F., Cangelli Filho, R., Cordás, T. A. et al. Síndromes psiquiátricas: diagnóstico e entrevista para profissionais de saúde mental. Porto Alegre: Artmed, 135-142.

Antoniuk, S. A. (2006). Desenvolvimento de um questionário breve para identificação do transtorno de déficit de atenção/hiperatividade direcionado a pais $e$ professores. 2006. 213 f. Tese (Doutorado em Saúde da Criança e do Adolescente) - Programa de PósGraduação em Saúde da Criança e do Adolescente no Departamento de Pediatria, Setor de Ciências da Saúde da Universidade Federal do Paraná, Paraná.

Asbahr, F. R., Costa, C. Z. G., \& Morikawa, M. Criança e adolescente. (2010). In: Neto, M. R. L. et. al. TDAH [transtorno de déficit de atenção/hiperatividade] ao longo da vida. Porto Alegre: Artmed, 146-160.

Benczik, E. B. P. (2000a). Manual da Escala de Transtorno de Déficit de Atenção/Hiperatividade (versão para professores). São Paulo: Casa do Psicólogo.

Benczik, E. B. P. (2000b). Transtorno de déficit de atenção/ hiperatividade: atualização diagnóstica e terapêutica, um guia de orientação para profissionais. São Paulo: Casa do Psicólogo.

Biederman, J., Mick, E., Faraone, S. V., Braaten, E., Doyle, A., Spencer,T., Wilens, T. E., Frazier, E., \& Johnson, M. A. (2002). Influence of Gender on Attention Deficit Hyperactivity Disorder in Children Referred to a Psychiatric Clinic. The American Journal of Psychiatry, v. 159 (1), 36-42. Recuperado em 14 de fevereiro de 2012, de http://ajp.psychiatryonline.org/article. aspx?articleID=175269

Biederman, J., Mick, E., \& Faraone, S. V. (2000). Agedependent decline of symptoms off attention deficit hyperactivity disorder: impact of remission definition and symptom type. The American Journal of Psychiatry, v. 157 (5), 816-818. Recuperado em 24 de fevereiro de 2012, de http://intl-ajp.psychiatryonline.org/data/Journals/AJP/3712/816.pdf
Caliman, L. V. (2008). O TDAH: entre as funções, disfunções e otimização da atenção. Psicologia em Estudo, Maringá, v.13 (3), 559-566.

Carreiro, L. R. R., Jorge, M., Tebar, M. R., Moraes, P. F., Araujo, R. R., Oliveira, T. A. E. R., \& Panhoni, V. A. C. S. (2008). Importância da interdisciplinaridade para avaliação e acompanhamento do transtorno do déficit de atenção e hiperatividade. Psicologia: Teoria $e$ Prática, v. 10 (2), 61-67.

Coutinho, G., Mattos, P., Schmitz, M., Fortes, D., \& Borges, M. (2009). Concordância entre relato de pais e professores para sintomas de TDAH: resultados de uma amostra clínica brasileira. Revista de Psiquiatria Clínica, v. 36 (3), 97-100.

Cypel, S. (2001). A criança com déficit de atenção e hiperatividade: atualização para pais, professores e profissionais da saúde. São Paulo: Lemos Editorial.

Eberstadt, M. (1999). Why ritalin rules. Policy Review, v. 94, 1-13. Recuperando em 08 de agosto de 2009, de http://www.hoover.org/publications/policyreview/3552192.html

Estados Unidos da América. Resolução 370 de 7 de junho de 2004. 108th Congress 2nd Session. 2004. Recuperado em 28 de fevereiro de 2012, de http:// www.govrecords.org/sres-370-rs-designating-september-7-2004-as.html

Faraone, S., Biederman, J., Spencer, T., Wilens, T., Seidman, L., Mick, E., \& Doyle, A. (2000). Attention-deficit/hiperactivity disorder in adults: an overview. Biological Psychiatry, (48), 9-20.

Folquito, C. T. (2009). Desenvolvimento psicológico e transtorno de déficit de atenção e hiperatividade (TDAH): a construção do pensamento operatório, 138 f. Dissertação (Mestrado em Psicologia) - Instituto de Psicologia da Universidade de São Paulo, São Paulo.

Gobbo, M. A., \& Neto, M. R. L. (2010). TDAH e gênero. In Neto, M. R. L. et. al. TDAH [transtorno de déficit de atenção/hiperatividade] ao longo da vida, 226-245.

Golfeto, J. H., Barbosa, G. A. (2003). Epidemiologia. In: Rohde, L. A., Mattos, P. et al. Princípios e práticas em TDAH. Porto Alegre: Artmed. 
Graeff, R., \& Vaz, C. E. (2008). Avaliação e diagnóstico do transtorno de déficit de atenção e hiperatividade (TDAH). Psicologia USP, São Paulo, v. 19, (3). Recuperado em 24 de fevereiro de 2012, de http:// www.revistasusp.sibi.usp.br/scielo.php?script=sci_ arttext\&pid=S1678-51772008000300005\&lng=pt\& nrm=iso

Kessler, R.C., Adler, L., Ames, M., Demler, O., Faraone, S., Hiripi, E., Howes, M.J., Jin, R., Secnik, K., Spencer, T., Ustun, T.B., \& Walters, E.E. (2005). The World Health Organization Adult ADHD Self-Report Scale (ASRS): a short screening scale for use in the general population. Psychological Medicine, v.35, 245-256. Recuperado em 28 de fevereiro de 2012, de http:// academicdepartments.musc.edu/psychiatry/research/cns/upadhyayareferences/Kessler_2005.pdf

Mattos, P., Palmini, A., Salgado, C. A., Segenreich, D., Grevet, E., Oliveira, I. R., Rohde, L. A, Romano, M., Louzã, M., Abreu, P. B., \& Lima, P. P. (2006). Painel brasileiro de especialistas sobre diagnóstico do tratamento do déficit de atenção/hiperatividade (TDAH) em adultos. Revista de Psiquiatria do Rio Grande do Sul, Porto Alegre, v. 28 (1), 50-60.

Mattos, P., Segenreich, D., Saboya, E., Louzã, M., Dias, G., \& Romano, M. (2006). Adaptação Transcultural para o português da escala Adult Self-Report Scale para avaliação do Transtorno de déficit de atenção/Hiperatividade (TDAH) em adultos. Revista de Psiquiatria Clínica, vol. 33 (4), 188-194.

Mattos, P., Serra-Pinheiro, M. A., Rohde, L. A., \& Pinto, D. (2006). Apresentação de uma versão em português para uso no Brasil do instrumento MTA-SNAP-IV de avaliação de sintomas de transtorno do déficit de atenção/hiperatividade e sintomas de transtorno desafiador e de oposição. Revista de Psiquiatria do Rio Grande do Sul [online]. vol. 28 (3), 290-297. ISSN 0101-8108.

Mesquita, R. C. (2009). A implicação do educador diante do TDAH: Repetição do discurso médico ou construção Educacional? 2009. 151 f. Dissertação (Mestrado em Educação) - Programa de Pós-Graduação da Faculdade de Educação da Universidade Federal de Minas Gerais, Belo Horizonte.
Organização Mundial da Saúde. (1993). Classificação de Transtornos Mentais e de Comportamento da CID-10: Descrições Clínicas e Diretrizes Diagnósticas. Artes Médicas, Porto Alegre.

Peixoto, A. L. B. (2006). “TDAH, quem realmente o tem?” Transtorno de déficit de atenção e hiperatividade na criança escolar: uso dos critérios diagnósticos e tratamento proposto pelos profissionais da saúde mental. Dissertação (Mestrado em Psicologia) - Programa de Pós-Graduação em Psicologia da Universidade Federal do Espírito Santo, Vitória.

Reinhardt, M. C. (2007). Avaliação da Resposta ao Tratamento com Metilfenidato em Pacientes com Transtorno de Déficit de Atenção/Hiperatividade com e sem Critério de Idade de Início de Sintomas antes dos 7 Anos. Dissertação (Mestrado em Medicina) - Programa de Pós-Graduação em Medicina: Psiquiatria da Universidade Federal do Rio Grande do Sul, Porto Alegre.

Rohde, L. A., Barbosa, G., Tramontina, S., \& Polanczyk, G. (2000). Transtorno de déficit de atenção/ hiperatividade, Revista Brasileira Psiquiatria, 22 (supl 2), 7-11.

Rohde, L. A., \& Halpern, R. (2004). Transtorno de déficit de atenção/hiperatividade: atualização. Jornal de Pediatria, 80 (supl 2), S61-S70.

Rossi, L.R.(2008).Efeitos de um programa pedagógico comportamental sobre TDAH para professores do ensino fundamental. Dissertação (Mestrado). Universidade Estadual Paulista "Júlio de Mesquita Filho - Psicologia do Desenvolvimento e Aprendizagem - Área de Concentração: Comportamento e Saúde.

Rotta, N. T., Ohlweiler, L., \& Riesgo, R. S. (2006). Transtornos da Aprendizagem: Abordagem Neurobiológica e Multidisciplinar. Porto Alegre: Artmed.

Schneider, H., \& Eisenberg, D. (2006). Who receives a Diagnosis of ADHD in the USA. Elementary School Population? Pediatrics, v. 117 (4), 601-609. Recuperado em 28 de fevereiro de 2102, de http:// pediatrics.aappublications.org/content/117/4/ e601.full.pdf+html

Vasconcelos, M. M., Malheiros, A. F. A., Werner, J. J., Brito, A. R., Barbosa, J. B., Santos, I. S. O., \& Lima, D. F. (2005). Contribuição dos Fatores de Risco Psicossociais para o TDAH. Arquivos de Neuropsiquiatria, 63(1), 68-44. 